\title{
The pathogenesis of spontaneous arterial dissection
}

Pathologists and surgeons recognise the presence of an intimal tear as a necessary component of aortic dissection as they see it in the postmortem room or operating theatre. The intimal tears often have surprisingly sharp rather than ragged edges and are transverse or vertical in relation to the long axis of the aorta rather than being oblique. A combination of transverse and longitudinal orientation may produce $T$ or cross shaped tears. The intimal tears occur in areas of the intima which are macroscopically normal-for example, they do not begin at the site of an atherosclerotic plaque. In contrast, as highlighted in a comprehensive review in this issue (pages 451-4), spontaneous coronary artery dissection is often not associated with an intimal tear. ${ }^{1}$ Such coronary dissections are, at least initially, subadventitial haematomas which compress the lumen from outside, causing acute myocardial ischaemia.

The distinction between aortic and coronary dissection with regard to intimal tears, however, may not be so stark. Some coronary dissections do develop an intimal tear, sometimes after apparently easy coronary arteriography carried out to investigate the cause of acute myocardial ischaemia. A coronary artery other than that which originally dissected may develop an intimal tear during angiography, suggesting a diffuse propensity for dissection rather than a purely local abnormality.

The question therefore arises whether aortic dissection also starts as an intramural haematoma which is followed by an intimal tear as a secondary event. Neither pathologists or surgeons are in a position to recognise this early phase. Modern imaging techniques, as in a report in this issue, ${ }^{2}$ can show that an intramural haematoma precedes the intimal tear in some cases. The computed tomogram clearly showed a translucent mass within the aortic wall which extended around three quarters of the circumference of the lumen. No intimal tear was seen. Back pain and cord ischaemia caused by compression of a lumbar artery had prompted the investigation. Subsequently, fully developed extending dissection with an intimal tear occurred.

Are all aortic dissections with intimal tears preceded by an intramural haematoma? The question cannot be answered; all that can be said is that because pathologists do not see organised healed haematomas in the aortic media at routine necropsy it would have to be postulated that virtually all intramural haematomas culminate in an intimal tear and fully developed dissection tracks.

It is credible that intramural haematomas could precipitate intimal tears. In the normal aortic wall in which the mechanical properties of the tissue are uniform, circumferential wall stress in systole is evenly distributed although there is a transmural gradient with the innermost layer of the media carrying most stress. Inhomogeneity of tissue characteristics leads to an uneven distribution of wall stress. Stress distribution across coronary plaques has been considered by computer modelling. ${ }^{3}$ The phenomenon applies equally to dissection of the aorta. A soft mass within the vessel wall, whether it be lipid or a haematoma, cannot carry tensile stress; the share of circumferential wall stress that it would have carried has to be redistributed elsewhere. The stress is redistributed to the innermost portion of the arterial wall over the soft mass. Focal increases in stress up to 10 times that exerted on the rest of the wall can occur particularly if the soft mass extends around a large arc of the vessel circumference and if the overlying tissue is stiffer than the rest of the vessel. Both conditions apply to intramural aortic haematomas. The case described on page 533 shows an intramural haematoma extending around an arc of more than $180^{\circ}$. With increasing age the aortic intima thickens and the whole aortic wall stiffens.

Why do intramural haematomas arise in the first instance? In the aorta there are up to 30 elastic laminae arranged in parallel and separated by smooth muscle cells. Small arterioles enter the aortic media and form an arcade of vessels running both transversely and longitudinally at a level 3-5 laminae in from the adventitia. It is in this plane that dissection occurs in the aorta. In contrast the coronary artery media is avascular and muscular and does not contain many elastic lamina. The plane of dissection in the coronary arteries lies between the adventitia and the media.

In the aorta the elastic laminae are in fact sheets of elastic tissue with fenestrations. There is potentially considerable shear force exerted in systole as these plates move in relation to each other. In the normal aorta, shear stress is kept to a minimum by the folded nature of the elastic sheets. Until the aorta is distended to the point that the elastic laminae are straight shear stress does not rise. Any tissue inhomogeneity in the media, whether it be focal fibrotic stiff areas or cystic spaces, negates this protection. Small intramural vessels that pass through fenestrations in the elastic laminae may be subjected to local large shearing deformities as neighbouring elastic plates move relative to each other. This deformation may cause arteriolar rupture leading to haematoma formation.

Between the elastic lamina are smooth muscle cells embedded in a collagen and proteoglycan rich matrix. Fibrillin is a $350 \mathrm{kD}$ glycoprotein which is abundant in the aortic wall. ${ }^{4}$ It localises to the ends of maturing elastic fibres and seems to be responsible for tissue organisation and adhesion and thus is important in resisting shear stresses. Many point mutations are now reported in the fibrillin gene on the long arm of chromosome 15 and are responsible for Marfan's syndrome. Both the aortic dilatation and dissection that occur in Marfan's syndrome are probably caused by a failure to resist shear forces within the media. In contrast genetic abnormalities of the collagen molecule itself, either type I (osteogenesis imperfecta) or type III (Ehlers-Danlos), lead to simple dilatation of the aorta. ${ }^{5}$ Rupture if it occurs is due to saccular aneurysms not dissection. Thus abnormalities of collagen itself reduce the ability of the arterial wall to resist expansile force but the tissue has normal resistance to shear stress. Hypertension would enhance the risk of both saccular aneurysms and dissection. 
In Marfan's syndrome there is often a structural change in the media that is recognisable by light microscopy. ${ }^{6}$ This takes the form of open spaces containing proteoglycans surrounded by fragmented elastic laminae (cystic medial necrosis). The change is often very focal which indicates that structural change due to the gene abnormality is a late phenomenon and that a reduction in the ability of the aorta to resist circumferential and shear stress occurs long before this stage. Any focal cystic change will, however, alter mechanical forces across the vessel wall and increase the risk of dissection.

A striking feature of Marfan's syndrome is the heterogeneity of the severity of the cardiovascular and skeletal expressions both between and within affected families. It is now clear that in part this is due to what is known as a dominant negative pathogenesis by the abnormal gene. ${ }^{57}$ The phenotypic expression depends on the presence and amount of the abnormal (mutant) fibrillin rather than being due to a simple deficiency of normal (wild) fibrillin. Families or individuals in which relatively little mutant fibrillin is produced are mildly affected. Families in which there is a premature termination codon on one copy of the fibrillin gene and which thus have a simple deficiency of normal fibrillin are also mildly affected. The poisoning effect of mutant fibrillin is due to its interference with the formation of multimers of fibrillin monomers which leads to inhibition of microfibril formation. The fibrillin gene is also responsible for some cardiovascular familial conditions which fall short of the criteria needed for designation as Marfan's syndrome. In mitral valve prolapse and mild aortic dilatation without dissection there is no progression to dissection and, other than a tendency to have long limbs, no skeletal disease. A fibrillin mutation has now been shown for this condition. ${ }^{8}$ It is very likely that familial aortic dissection without other stigmata of Marfan's syndrome is also going to have defects in fibrillin.

There have been major advances in our understanding of Marfan's syndrome, however, most subjects with dissection of the aorta have nothing to suggest they have a familial systemic gene defect. The major risk factor is hypertension and there is also an association with bicuspid. aortic valves, which are found in up to $1 \%$ of the general population. ${ }^{9}$

In both hypertension and increasing age the uniformity of the medial structure is lost. ${ }^{10}$ Zonal fibrosis replacing the smooth muscle and elastic tissue occurs in focal areas. Cystic breakdown of elastic lamellae also occurs as an age related change. Though these changes are focal they potentially lead to major changes in the distribution of both circumferential wall stress and shear stress in the media and predispose to dissection.

In bicuspid aortic valves it is tempting to ascribe dilatation of the ascending aortic and risk of dissection to purely mechanical causes attributable to the abnormal flow in the aorta. There is, however, little correlation between the degree of valve dysfunction, high pressure jet flow, or regurgitation and the degree of aortic dilatation. ${ }^{11}$ It therefore remains possible that an abnormality of a gene controlling both the development of the aortic valve and the aortic media is responsible. The recent recognition of abnormalities in the gene controlling tropelastin in supraaortic stenosis ${ }^{12}$ supports this view.

Coronary artery dissection while having some morphological and mechanistic similarities to aortic dissection seems to be pathogenetically distinct. There is no relation to Marfan's syndrome, no family history, and no clear link with hypertension. For some reason the subjects acquire a loosening of adhesion of the media to the adventitia. Some cases are related to pregnancy in which a systemic increase in tissue laxity does occur. A recurrent theme of pathology reports is an infiltrate of basophils and eosinophils in the adventitia, although this falls far short of what could be designated an arteritis. There is no necrosis of the media or evidence of antigen-antibody complexes. The inflammatory cells, in particular basophils, could, however, produce metalloproteinases capable of weakening the connective tissue matrix. One feature of dissection of the coronary arteries is that the episodes are often multiple. Nevertheless the tendency to dissect diminishes in subjects who survive. Another feature is that where no intimal tear develops the artery can revert to being angiographically normal. Coronary dissection is therefore one explanation of the clinically observed phenomenon of normal angiograms in a young person, often female, who suffers an acute regional infarct.

Cardiovascular Pathology,

M J DAVIES

St George's Hospital Medical School,

Cranmer Terrace,

London SW17 ORE

Cardiovascular Surgery,

T TREASURE

St George's Hospital Medical School, London

Department of Engineering and Physiology,

P D RICHARDSON

Brown University,

Rhode Island,

USA

1 Basso C, Morgagni GL, Thiene G. Spontaneous coronary artery dissection: a neglected cause of acute myocardial ischaemia and sudden death. tion: a neglected cause

2 Ferguson J D, Moore N, Banning AP. Intramural aortic haematoma causing ischaemia of the spinal cord. Heart 1996;75:533.

3 Richardson PD, Davies MJ, Born GVR. Influences of plaque configuration and stress distribution on fissuring of coronary atherosclerotic plaques. Lancet 1989;ii:941-4.

4 Pereira L, Levran O, Ramirez F, et al. A molecular approach to the stratification of cardiovascular risk in families with Marfan's syndrome. $N$ Engl $\mathcal{F}$ Med 1994;331:148-53.

5 Dietz HC. New insights into the genetic basis of aortic aneurysms. In: Schoen FJ, Gimbrone MA, eds. Cardiovascular pathology, clinicopathologic correlations and pathogenetic mechanisms. Baltimore: Williams \& Wilkins; 144-55.

6 Niitsuya M, Kuwao S, Sato B, Kameya T, Kikawada R. Histopathological study of aortic wall disection. $\mathcal{F}$ Cardiol 1991;21:445-52.

7 Herskowitz I. Functional inactivation of genes by dominant negative mutations. Nature 1987;329:219-22.

8 Dietz HC, McIntosh I, Sakai LY, et al. Four novel FBN1 mutations: signifcance for mutant transcript level and EGF-like domain calcium binding in the pathogenesis of Marfan syndrome. Genomics 1993;17:468-75.

9 Edwards J. The congenitally bicuspid aortic valve. Circulation 1961;23: 485-8.

10 Schlatmann T, Becker A. Pathogenesis of dissecting aneurysms of aorta. Comparative histopathologic study of significance of medial changes. $A m$ 7 Cardiol 1977;39:21-6.

11 Hahn RT, Roman MJ, Mogtader AH, Devereux RB. Association of aortic dilatation with regurgitant, stenotic and functionally normal bicuspid dilatation with regurgitant, stenotic and function
aortic valves. $\mathcal{F}$ Am Coll Cardiol 1992;19:283-8.

12 Ewart EK, Morris CA, Atkinson D, et al. Hemizygosity at the elastin locus in a development disorder. William's syndrome. Nature Genet 1993;3: 